\title{
A Survey of the Application of Rough Sets in Intelligent Decision Support System
}

\author{
Yehong Han \\ School of Information Science and Engineering, University of Qilu Normal, Jinan 250014, China \\ sdzzhyh@163.com
}

Keywords: Rough sets, Intelligent decision support system, Knowledge acquisition, Uncertain reasoning.

Abstract. Intelligent decision support system which can help to make decision in solving the complex decision problems through logical reasoning is the combination of decision support system and artificial intelligence technology. Rough sets technology is a mathematical tool for data analysis to deal with fuzzy and uncertainty information. The latest researches of two applications of rough sets in intelligent decision support system which include knowledge acquisition and uncertain reasoning are reviewed.

\section{Introduction}

There are varying degrees of uncertainty in many practical application systems. The collected data often contain noise, inaccuracy and infirmity. Following probability theory, fuzzy set and evidence theory, rough set theory is a novel mathematical tool to deal with uncertainty which include classification of fuzzy, inaccurate or incomplete information. The decision rules and classification definition can be obtained through the reduction of knowledge with preserving the classification ability of information system unchanged. Rough set theory is based on the classification mechanism.

Rough set theory regards classify as equivalence relations on a particular space. The equivalence relations constitute a division of the relation space. The difference of rough set and another theory which deal with uncertainty and imperfection is that rough set does not require any prior information beyond the data collection process. Recent years witness the prosperity of intelligent decision support

system (IDSS) with the development of artificial intelligence and knowledge engineering application. Intelligent decision support system is the combination of artificial intelligence and decision support system. Based on expert system technology, decision support system can fully use decision-making descriptive knowledge, decision-making process of procedural knowledge and reasoning knowledge for solving problem.

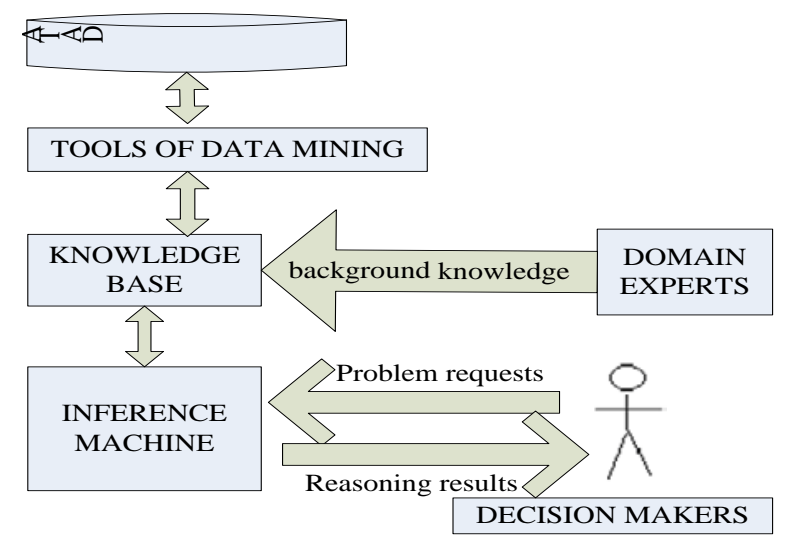

Fig. 1 The frame of IDSS based on data mining 


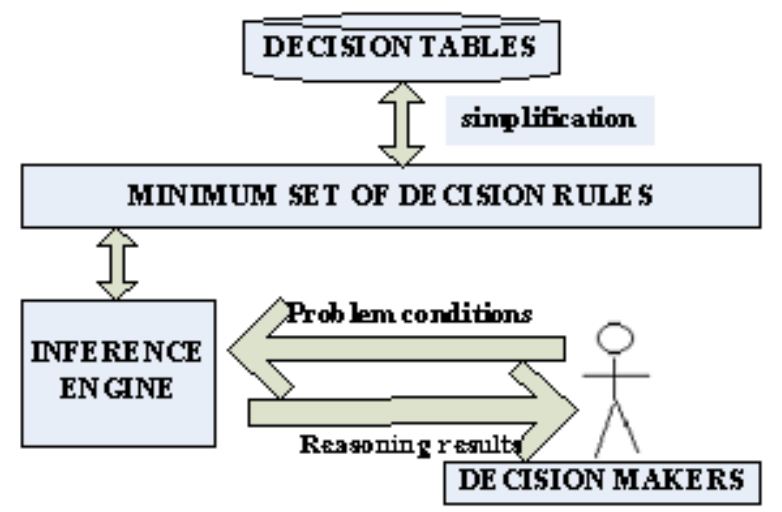

Fig. 2 The frame of IDSS based on rough sets

The frame of intelligent decision support system based on technology of data mining is shown in Fig.1. Based on data mining technologies which can discover previously unknown and potentially useful information from large databases, decision ability of intelligent decision support system can be improved greatly. But knowledge accessed from database of decision domain are often uncertainty and incomplete, and decision making based on the uncertainty or incomplete knowledge is a difficulty. Exactly, rough set is suit for dealing with uncertainty and vagueness information. The frame of IDSS based on rough sets theory is shown in Fig.2. The data structure of the knowledge induced from empirical data is regarded as records of objects described by a set of attributes. The knowledge is understood as an ability to classify objects. In the frame of IDSS based on tough sets, there are different types of applications which include knowledge acquisition, uncertain reasoning, knowledge update and so on. The rest of this paper reviews two kinds of applications which include knowledge acquisition and uncertain reasoning.

\section{Knowledge acquisition}

Based on rough sets theory, knowledge acquisition in intelligent decision support system can be seen as the simplification of knowledge representation system. The association of knowledge representation and knowledge acquisition is shown below in Fig.3. Knowledge representation system can be formulated as a pair $S=(U, A)$ among which $U$ is a nonempty and finite set called the universe, $A$ is a nonempty and finite set of primitive attributes. Primitive attribute $a \in A$ is a function $a: U \rightarrow V a$. If $u \in U, a \in A$ and $v_{a} \in V a$, then knowledge representation system will been called complete knowledge representation system, otherwise incomplete knowledge representation system. $C, D$ is called as condition and decision attributes respectively, let $C, D \subset A$, knowledge representation system with distinguished condition and decision attributes will be denoted $S=(U, A, C, D)$.

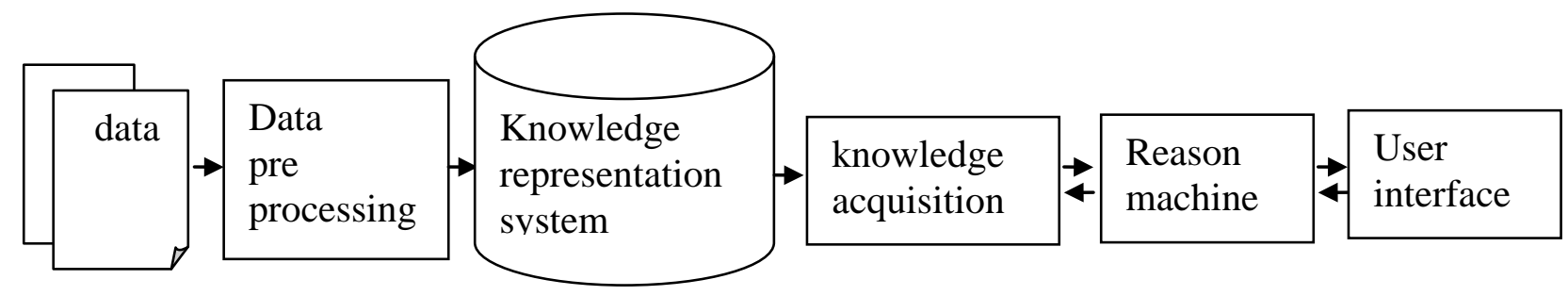

Fig.3 The association of knowledge representation and knowledge acquisition

$S=(U, A, C, D)$ can represent a knowledge representation system. When $P O S_{C-\{a\}}(D)=P O S_{C}(D)$, a which is an attribute is dispensable in knowledge representation system, otherwise a is indispensable. If all of the attributes $a \in C$ are indispensable, knowledge representation system is independent. 
Let $R \subseteq C, P O S_{R}(D)=P O S_{C}(D)$ and $S^{\prime}=(U, R \cup D, R, D)$ is independent, $R$ which can be denoted $R E D(C, D)$ is a reduction of condition attributes. $S$ ' is the reduction of $S=(U, A, C, D)$. The set which includes all of indispensable attributes in condition attributes can be denoted $\operatorname{CORE}(C, D)$. What's more, $\operatorname{CORE}(C, D)=\bigcap \operatorname{RED}(C, D)$. Słowiński [1] refers to states or examples of a decision situation as objects. The knowledge discovery paradigm for multi-attribute and multi-criteria decision making is presented. The Object with the same description can be seen similar with respect to the available information. All of the indiscernible relations constitute the mathematical basis of rough sets which induce a partition of the universe into blocks of indiscernible objects, called elementary sets. The goal of feature selection which can be called attribute reduction is retaining the discriminatory of original features. In the paper [2], a theoretic framework called positive approximation is presented so as to accelerate the heuristic process of attribute reduction. An accelerator attribute reduction algorithm which enhances representative heuristic attribute reduction is produced. The basic vector $H(X)$ denoted by $\mathrm{H}[\mu, v](X), \mathrm{H}(\mu, v](X), \mathrm{H}[\mu, v)(X)$ and $\mathrm{H}(\mu, v)(X)$ is induced from the relation matrix based on a matrix representation of rough set approximations[3]. The algorithm corresponding to the incremental approaches is presented. The incremental approaches whose goal is to update relation matrix can update rough set approximations. Rule-base evidential reasoning is proved to be a valuable to build intelligent decision support systems. Nevertheless, there are two defects which reduce their ability to deal with uncertainties. Firstly, the degree of belief can be assigned only to a particular hypothesis, not to a group of them, whereas an assignment of a belief mass to a group of events is a key principle of the Dempster-Shafer theory. Secondly, limitation is concerned with the observation that in many decisions we deal with different sources of evidence and the combination of them is needed. Traditional rule-base evidential reasoning can not combine evidence among different sources. Dymova et al.[4] introduce a novel approach whose advantages are demonstrated using simple numerical examples and the developed stock trading expert system optimized and tested on the real data from Warsaw Stock Exchange. Traditional rough sets method focus on the approximation of sets described by a single equivalence relation. Classical rough-set theory is based on a single granulation from granular computing point of view.

\section{Uncertain reasoning}

Uncertainty is the essential characteristic of intelligent questions. Intelligent is mainly reflected by the ability to deal with uncertainty problem. Uncertainty reasoning is an important research area in intelligent decision support system. Based on above description on the association of knowledge representation and knowledge acquisition, $\alpha \rightarrow \beta$ which is a implication is called as a decision rule and denoted $r$ in the rough set theory; $\alpha$ and $\beta$ which are formula of the form $\left(a_{1}, v_{1}\right) \wedge\left(a_{2}, v_{2}\right) \wedge \ldots \wedge\left(a_{n}, v_{n}\right)$ are referred to as the predecessor and the successor of $\alpha \rightarrow \beta$ respectively, where $a_{i}(i=1,2, \ldots, n)$ is a attribute of a decision table and $v_{i}(i=1,2, \ldots, n)$ is the value of the attribute $a_{i}$.

All of possible conclusion should be given a weight if the conclusions of new object in the process of uncertainty reasoning want to be acquired. Uncertainty reasoning technologies include object tracking, attribute voting, complete matching and so on. The set of decision rules are obtained through simplification of a decision table. Above set can be denoted RUL. When $x$ is a new object and there is a decision rule $\alpha \rightarrow \beta$ satisfying $x \rightarrow \alpha$, the rule $\alpha \rightarrow \beta$ may be called matching the new object $x$. The matching set consists of all decision rules which match the new object $x$, and will be denoted $R U L(x)$. 


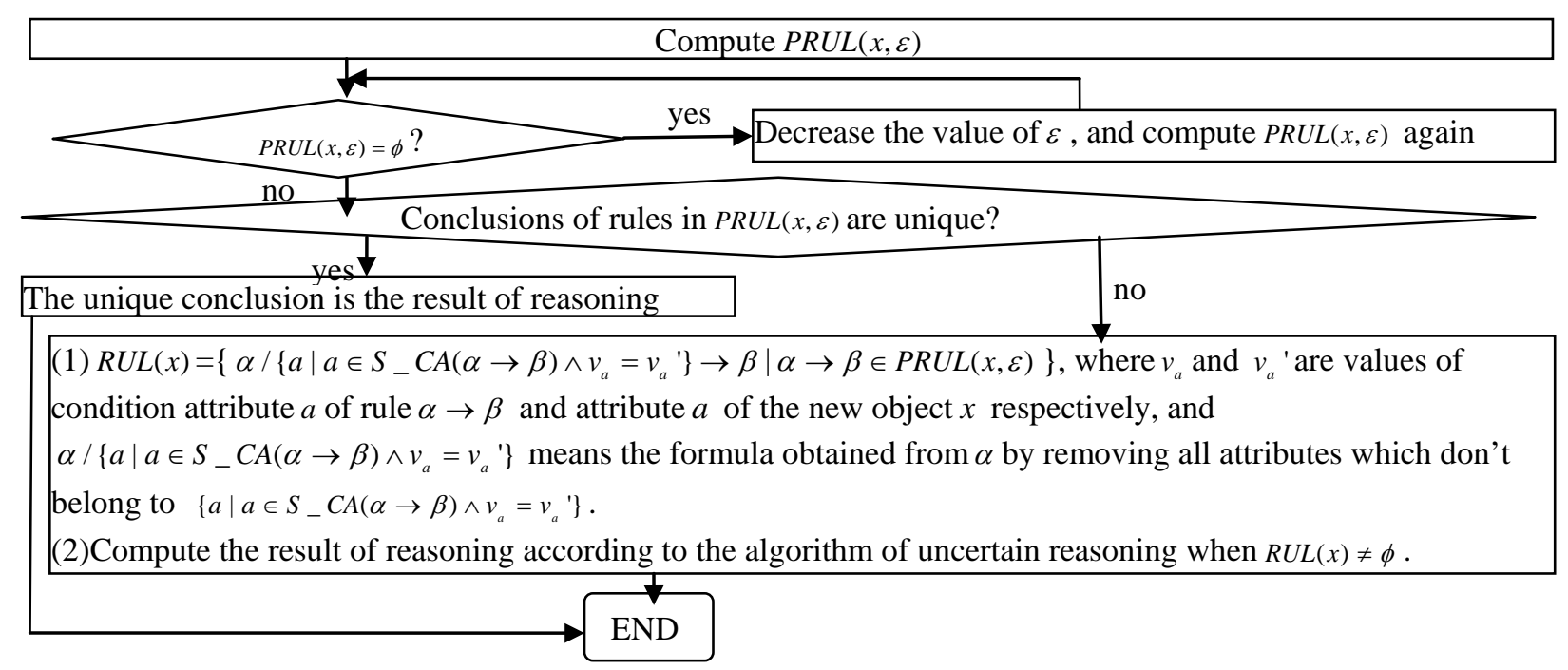

Fig.4 The algorithm of uncertain reasoning considering subjective factors when $\operatorname{RUL}(x)=\phi$

An algorithm of uncertain reasoning considering subjective factors when $R_{L L}(x)=\phi$ is shown in Fig.4. Above algorithm of uncertain reasoning considering subjective factors overcomes shortcomings of traditional uncertain reasoning methods and the result of uncertain reasoning is more suitable for different demands. But there are two restrictive conditions in the process of reasoning using the algorithm of uncertain reasoning considering subjective factors. Firstly, the object $x$ should satisfy $s_{-} C A(x) \supseteq \bigcup_{r \in m u} s_{-} C A(r)$. Secondly, the degree of significance with $a$ of the new object must satisfy $0<\operatorname{Sig}(a)<1$. The two restrictive conditions mean that all attributes of the new object are useful in the process of reasoning.

In the paper [5], rough sets model based on a tolerance relation to an incomplete rough sets model based on multi granulations is enlarged. Set approximations are defined through using multiple tolerance relations on the universe. Approximation reduction is presented in order to characterize the smallest attribute subset which preserves the lower approximation and upper approximation. Huang B et al. [6] introduce a dominance-based rough set model based on the substitution of the indistinguishable relation by a dominance relation that is defined on the score and accuracy function of intuitional fuzzy value. Based on concept algebra, a formal knowledge representation system which uses the object attribute relation model for knowledge representation for autonomous concept formation is presented in the paper [7]. Above models include architectural model, behavioral model and conceptual model. The frame can be regarded as a component towards the development of intelligent decision support system in cognitive computing and computational intelligence

\section{Summary}

Recently, rough sets technology has been widely used in a variety of domains which include decision support system, machine learning, expert systems and pattern recognition. In the frame of intelligent decision support system based on tough sets, there are different types of applications which include knowledge acquisition, uncertain reasoning and knowledge update. This paper reviews the latest researches of knowledge acquisition and uncertain reasoning. We strongly believe that this research field will be paid more and more attentions and will promote the fundamental theories research in the related fields.

\section{Acknowledgement}

In this paper, the research was sponsored by the Natural Science Foundation of Shandong Province, China (Grant No. ZR2013FL010). 


\section{References}

[1] Słowiński R, Greco S, Matarazzo B, Rough-set-based decision support, Search Methodologies, 557-609, 2014.

[2] Qian Y, Liang J, Pedrycz W, Positive approximation: an accelerator for attribute reduction in rough set theory, Artificial Intelligence, 174(9) 597-618, 2010.

[3] Zhang J, Li T, Ruan D, Rough sets based matrix approaches with dynamic attribute variation in set-valued information systems, International Journal of Approximate Reasoning, 620-635, 2012.

[4] Dymova L, Sevastianov P, Bartosiewicz P, A new approach to the rule-base evidential reasoning: Stock trading expert system application, Expert systems with Applications, 37(8) 5564-5576, 2010.

[5] Qian Y, Liang J, Dang C. Incomplete multigranulation rough set, Systems, Man and Cybernetics, Part A: Systems and Humans, IEEE Transactions on, 40(2) 420-431, 2010.

[6] Huang B, Li H, Wei D, Dominance-based rough set model in intuitionistic fuzzy information systems, Knowledge-Based Systems, 24(1) 115-123, 2012.

[7] Tian Y, Wang Y, Gavrilova M L, A formal knowledge representation system (FKRS) for the intelligent knowledge base of a cognitive learning engine, International Journal of Software Science and Computational Intelligence, 3(4) 1-17, 2011. 